\title{
Tratamiento de las estenosis laríngeas adquiridas en pediatría. Serie de casos
}

\author{
Treatment of acquired laryngeal stenosis in pediatrics. Case series
}

\author{
Dra. Giselle Cuestas ${ }^{a}$, Dr. Mauricio Urquizo ${ }^{b}$, Dra. Victoria Demarchi ${ }^{a}$, Dr. Adrián Zanetta ${ }^{a}$, Dr. Pablo Lobos ${ }^{b}$ \\ y Dr. Juan Razetti ${ }^{a}$
}

\section{RESUMEN}

La estenosis subglótica es una de las causas más frecuentes de obstrucción de la vía aérea superior en los niños. Si bien puede tener un origen congénito, la mayoría de las estenosis son adquiridas. Debe pensarse en esta patología en todo niño con antecedentes de intubación, instrumentación o trauma de la vía aérea que presenta dificultad respiratoria.

El diagnóstico se sospecha por la clínica, los antecedentes y la radiografía cervical, y se confirma mediante el examen endoscópico. La conducta terapéutica dependerá, entre otros factores, del grado de estenosis que se presente.

Describimos nuestra experiencia con 6 pacientes que presentaban estenosis subglótica posintubación, tratados quirúrgicamente con técnica de expansión.

Palabras clave:estenosis subglótica, niños, intubación endotraqueal, tratamiento quirúrgico.

\begin{abstract}
Subglottic stenosis is one of the most common causes of upper airway obstruction in children. Even though it may have a congenital origin, most of them are acquired stenosis. This condition should be suspected in any child with a history of intubation, instrumentation or trauma of the airway that is having difficulty breathing.

The diagnosis is suspected by clinical, history and cervical radiograph, and is confirmed by endoscopic examination. Among others factors the treatment depends on the stenosis degree.

We describe our experience with 6 patients with post-intubation subglottic stenosis treated surgically with expansion technique. Keywords: subglottic stenosis, children, endotracheal intubation, surgical treatment.
\end{abstract}

http:/ /dx.doi.org/10.5546/aap.2013.e136

\section{INTRODUCCIÓN}

La estenosis subglótica es la disminución del calibre de la laringe por debajo de las

a. Servicio de Otorrinolaringología Infantil.

b. Servicio de Cirugía Pediátrica.

Hospital Italiano de Buenos Aires.

Correspondencia:

Dra. Giselle Cuestas: giselle_cuestas@yahoo.com.ar

Conflicto de intereses: Ninguno que declarar.

Recibido: 21-6-2013

Aceptado: 24-7-2013 cuerdas vocales. Más del $90 \%$ de las estenosis son adquiridas; la intubación endotraqueal es la causa principal. ${ }^{1-6} \mathrm{La}$ incidencia de estenosis posintubación es de $0,9 \%$ a $3 \% .{ }^{2}$ Es más frecuente en la infancia porque la subglotis es la zona más estrecha de la vía aérea pediátrica y está rodeada en su totalidad por cartílago (el anillo cricoides). ${ }^{7}$

Se presenta como un cuadro obstructivo laríngeo de gravedad variable. Puede manifestarse con fracaso en la extubación o con la necesidad de reintubar con un tubo endotraqueal más pequeño. En otros pacientes, la sintomatología de obstrucción respiratoria alta se inicia después de unos días de haber sido extubados (dentro de los 21 días) conforme progresa la fibrosis. ${ }^{7}$ Los síntomas principales son la disnea, el estridor bifásico y el tiraje. Puede acompañarse de llanto débil o disfonía.

La radiografía cervical en hiperextensión con técnica de partes blandas, anteroposterior y perfil permite visualizar la estenosis. La tomografía computarizada con reconstrucción tridimensional de la vía aérea y la resonancia magnética proporcionan información precisa de la localización y extensión de la estenosis, y son de gran utilidad para plantear la estrategia quirúrgica. En todos los casos, debe realizarse una endoscopia flexible bajo anestesia tópica para descartar una enfermedad glótica o supraglótica, como parálisis de cuerdas vocales y laringomalacia. El diagnóstico se confirma en el quirófano con una endoscopia rígida bajo anestesia general, colocando un laringoscopio de comisura que separe delicadamente las cuerdas vocales.

El manejo de esta patología en los niños es complejo. Se ha diseñado una amplia variedad de técnicas quirúrgicas y endoscópicas. La reconstrucción con técnica de expansión del marco laríngeo mediante la división del cricoides y la colocación, por lo general, de un injerto de cartílago, ha demostrado ser una técnica eficaz para el manejo quirúrgico de la estenosis. Describimos nuestra experiencia con esta técnica. 


\section{CASOS CLÍNICOS}

Presentamos a 6 pacientes con estenosis subglótica posintubación operados en la Unidad de Vía Aérea Pediátrica formada por los Servicios de Otorrinolaringología Infantil y Cirugía Pediátrica del Hospital Italiano de Buenos Aires, entre noviembre de 2010 y abril de 2013.

Las características de los pacientes, incluidos sexo, causa de la estenosis, grado de la lesión (clasificación de Cotton), edad en el momento de la cirugía, tratamientos previos, técnica quirúrgica utilizada y comorbilidades, se resumen en la Tabla 1.

Todos los pacientes fueron valorados con endoscopia con anestesia local (con fibra óptica flexible) para evaluar la indemnidad de las estructuras supraglóticas (movimiento de cuerdas vocales y aritenoides) y con anestesia general (con instrumental rígido) para determinar las características de la lesión: grado, tipo, localización y extensión de la estenosis (Figura 1). También se realizó una tomografía computarizada con reconstrucción tridimensional de la vía aérea (Figura 2) y evaluación multidisciplinaria para valorar el reflujo gastroesofágico, los trastornos deglutorios y la enfermedad bronquial recurrente.

Todos los pacientes estaban traqueotomizados en el momento de la cirugía. En 2 pacientes se realizaron procedimientos endoscópicos previos a la cirugía abierta. La edad media al momento de la cirugía fue de 2,8 años.

El procedimiento quirúrgico consistió en una laringotraqueofisura con resección de la cicatriz subglótica, en 4 casos con interposición anterior y posterior de injerto costal y en 1 caso con inserción sólo anterior del injerto. En 4 pacientes se colocó un tubo en T de Montgomery y, en los restantes, un tubo endotraqueal (Figura 3).

En el posoperatorio se indicó tratamiento antirreflujo, antibióticos, analgésicos y alimentación por sonda nasogástrica hasta probar la tolerancia oral. El promedio de internación fue de 11 días.

Las complicaciones posquirúrgicas incluyeron

TABLA 1. Pacientes con estenosis subglótica adquirida

\begin{tabular}{|c|c|c|c|c|c|c|c|}
\hline $\mathbf{P}$ & S & $\begin{array}{c}\text { Causa de } \\
\text { la estenosis }\end{array}$ & $\begin{array}{l}\text { Clasificación } \\
\text { de Cotton }\end{array}$ & $\begin{array}{l}\text { Procedimientos } \\
\text { previos }\end{array}$ & $\begin{array}{c}\text { Edad al momento } \\
\text { de la cirugía }\end{array}$ & Tratamiento & Comorbilidades \\
\hline 1. & M & $\begin{array}{c}\text { TCE } \\
\text { AVM } 2 \text { meses }\end{array}$ & Grado III & Traqueotomía & 9 meses & $\begin{array}{l}\text { RLT con TM por } \\
6 \text { meses }\end{array}$ & $\begin{array}{c}\text { Convulsiones } \\
\text { Metabolopatía } \\
\text { BOR }\end{array}$ \\
\hline 2. & $\mathrm{M}$ & $\begin{array}{c}\text { DBP } \\
\text { AVM } 4 \text { meses }\end{array}$ & Grado IV & Traqueotomía & 6 años & $\begin{array}{c}\text { RLT con injerto } \\
\text { ant y TM por } 6 \text { meses }\end{array}$ & DBP \\
\hline 3. & $\mathrm{~F}$ & $\begin{array}{c}\text { DBP } \\
\text { AVM } 3 \text { meses }\end{array}$ & Grado III & Traqueotomía & 4 años & $\begin{array}{l}\text { RLT con injerto ant y } \\
\text { post y TM por } 6 \text { meses }\end{array}$ & $\begin{array}{l}\text { DBP } \\
\text { RGE }\end{array}$ \\
\hline 4. & $\mathrm{~F}$ & $\begin{array}{l}\text { Neumonía } \\
\text { AVM } 1 \text { mes }\end{array}$ & Grado III & Traqueotomía & 2 años & $\begin{array}{l}\text { RLT con injerto ant y } \\
\text { post y TET por } 8 \text { días }\end{array}$ & $\begin{array}{c}\text { CHARGE } \\
\text { Parálisis facial }\end{array}$ \\
\hline 5. & $\mathrm{~F}$ & $\begin{array}{c}\text { SALAM } \\
\text { AVM } 1 \text { mes }\end{array}$ & Grado III & $\begin{array}{c}\text { Traqueotomía } \\
\text { Dilatación laríngea }\end{array}$ & 1 año & $\begin{array}{l}\text { RLT con injerto ant y } \\
\text { post y TET por } 8 \text { días }\end{array}$ & $\begin{array}{l}\text { RGE } \\
\text { BOR }\end{array}$ \\
\hline 6. & M & $\begin{array}{c}\text { DBP } \\
\text { AVM } 3 \text { meses }\end{array}$ & Grado IV & $\begin{array}{c}\text { Traqueotomía } \\
\text { Dilatación laríngea y } \\
\text { colocación de TM }\end{array}$ & $\begin{array}{l}3 \text { años y } \\
6 \text { meses }\end{array}$ & $\begin{array}{l}\text { RLT con injerto ant y post } \\
\text { y TM por } 6 \text { meses* }\end{array}$ & $\begin{array}{c}\text { DBP } \\
\text { Traqueomalacia } \\
\text { Neurofibromatosis 1 } \\
\text { Gastrostomía }\end{array}$ \\
\hline
\end{tabular}

P: pacientes; S: sexo; M: masculino; F: femenino; TCE: traumatismo craneoencefálico; AVM: asistencia ventilatoria mecánica; DBP: displasia broncopulmonar; SALAM: síndrome de aspiración de líquido amniótico meconial; RLT: reconstrucción laringotraqueal; TM: tubo en T de Montgomery; Ant: anterior; Post: posterior; TET: tubo endotraqueal; BOR: bronquitis obstructiva recurrente; RGE: reflujo gastroesofágico.

*El paciente cursa el segundo mes con la prótesis. 
granulomas en el extremo proximal del tubo en $\mathrm{T}$ (caso 2) y granulomas laríngeos después de la extracción de la prótesis (casos 2 y 3). En ambos pacientes, los granulomas se resecaron por vía endoscópica.

El tiempo medio de seguimiento poscirugía fue de 14 meses. Se logró la decanulación en 4 pacientes (casos 1, 3, 4 y 5), con buena ventilación, deglución y fonación. Un paciente (caso 2) presentó recurrencia de la estenosis durante

FIGURA 1. Imagen endoscópica de estenosis subglótica grado III de Cotton

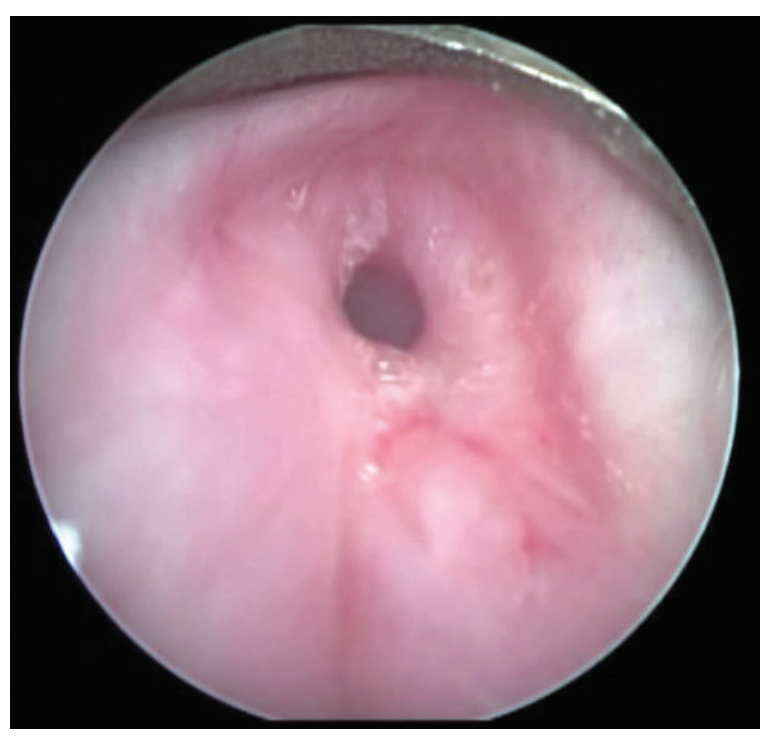

el seguimiento alejado, por lo que requiere cirugía de revisión, y el restante paciente (caso 6) permanece con el tubo en $\mathrm{T}$.

\section{DISCUSIÓN}

La estenosis subglótica adquirida es una de las causas más comunes de obstrucción de la vía aérea. A diferencia de las congénitas, es secundaria a un procedimiento médico y su tratamiento siempre es más dificultoso. ${ }^{7}$
FIgURA 2. Estenosis subglótica. Tomografía computada. A. Corte sagital. B. Endoscopia virtual. C. Reconstrucción tridimensional. D. Corte coronal

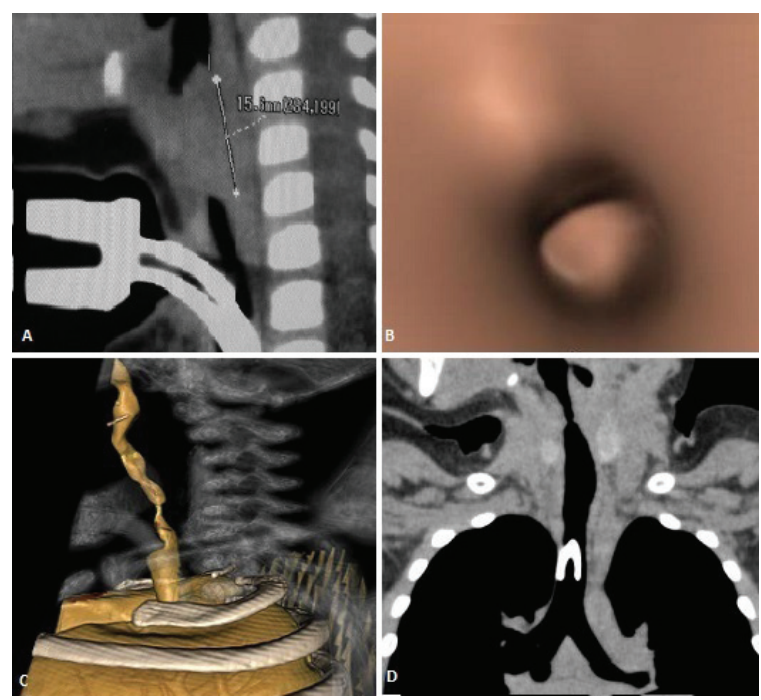

Figura 3. Imágenes intraoperatorias. A. Colocación de injerto costal. B. Injerto costal anterior en posición. C. Tubo en $T$ de Montgomery en posición

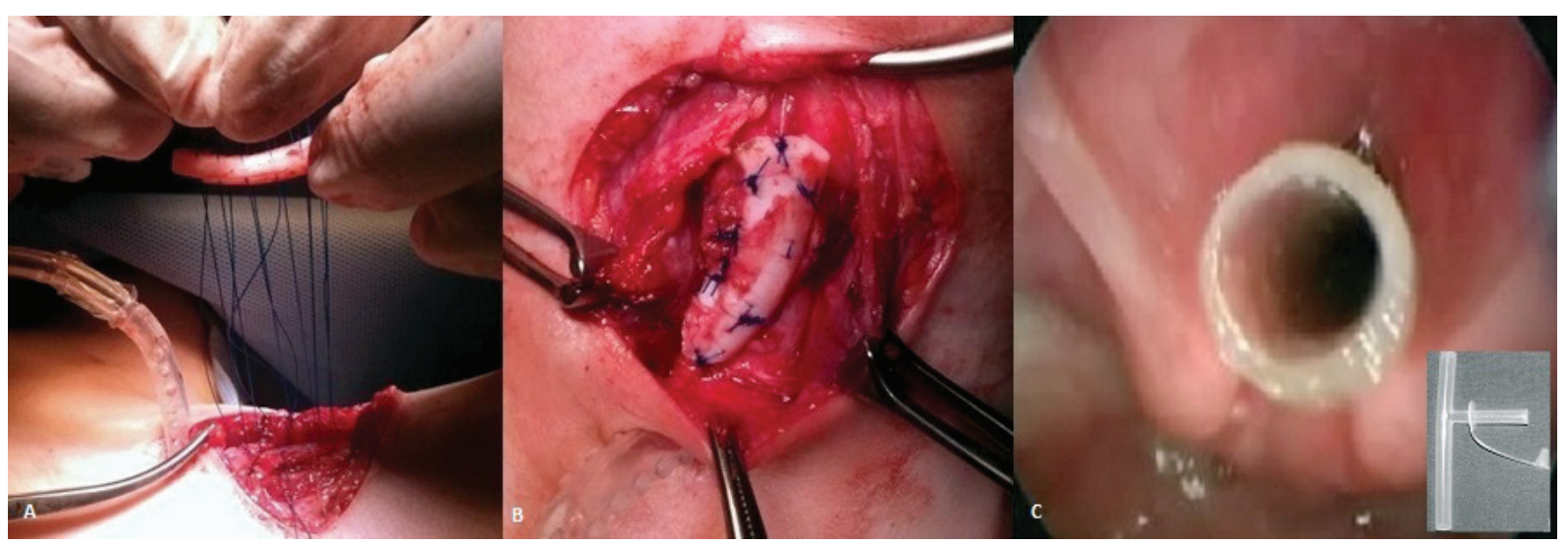


La causa más frecuente es la intubación. La intubación traumática y la presión inducida por el tubo son los principales factores que contribuyen a la estenosis posintubación. ${ }^{2}$ Cuando la presión del tubo excede la presión de perfusión capilar causa isquemia, seguida de edema, necrosis y úlceras. Posteriormente, en el proceso de reparación, el tejido de granulación puede ocasionar disminución de la luz de la vía aérea y obstrucción. ${ }^{1}$ Se considera que más allá de las 4 semanas de intubación el riesgo de estenosis se incrementa por la sobreinfección, pero el daño es posible, incluso, dentro de las 48 horas. $^{2}$

Otras etiologías traumáticas de menor incidencia son las quemaduras térmicas y químicas, las infecciones, el reflujo gastroesofágico, los traumatismos externos, las lesiones quirúrgicas secundarias a cirugías laríngeas previas y la realización de una traqueotomía alta, a nivel del anillo cricoides. ${ }^{5}$

La estenosis subglótica fue clasificada por Robin Cotton en cuatro grados según el porcentaje de luz comprometido (grado I: obstrucción de hasta el 50\%, grado II: hasta el 70\%, grado III: hasta el 99\%, grado IV: sin luz perceptible). ${ }^{4,8}$

La conducta terapéutica depende del grado y la longitud de la estenosis, y del estado general del paciente. Varía desde una conducta expectante en los grados leves hasta las cirugías complejas en las estenosis graves. No hay una única modalidad terapéutica para todos los casos de estenosis. El tratamiento debe ser individualizado. Las estenosis graves aumentan la probabilidad de que sea necesario más de un procedimiento para lograr la mejoría de la luz de la vía aérea y la decanulación.

Es importante realizar un estudio de reflujo gastroesofágico. Está demostrada la influencia negativa de este en las estructuras laringotraqueales y puede hacer fracasar el tratamiento, por lo que debe tratarse enérgicamente (a veces quirúrgicamente) antes de la cirugía. ${ }^{4}$

El tratamiento endoscópico (dilatación con bujías o balón, láser de $\mathrm{CO}_{2}$ ) es apropiado para la estenosis subglótica de grado I sintomática y algunos casos de grado II. Para los grados III y IV se requieren procedimientos más complejos. ${ }^{5}$ Se ha diseñado una amplia variedad de técnicas quirúrgicas, entre ellas, la reconstrucción laringotraqueal y la resección cricotraqueal. ${ }^{6}$

La reconstrucción laringotraqueal con técnica de expansión de la vía aérea mediante la colocación de un injerto de cartílago (costal o ala tiroidea) en la pared anterior o posterior del cricoides tiene como objetivo ampliar la zona estrechada hasta alcanzar un calibre semejante al normal. ${ }^{9}$ Se ha convertido en el tratamiento estándar de la estenosis subglótica moderada a grave en la edad pediátrica. Puede realizarse en uno o dos tiempos. En dos pacientes (casos 4 y 5) se efectuó en un tiempo, lo que permitió evitar largos períodos de prótesis endoluminal.

El injerto puede ser anterior (en estenosis anterior o colapso de pared anterior) o posterior (en estenosis posterior o extensión glótica). ${ }^{9} \mathrm{Si}$ bien la interposición del injerto no es siempre necesaria para obtener una expansión estable, su uso mejora la reepitelización y reduce la contracción cicatrizal. ${ }^{5}$

Las prótesis endoluminales proveen estabilidad a la vía aérea reconstruida. ${ }^{10-12}$ Las más utilizadas son las de silicona y la más conocida es el tubo en T de Montgomery. Este tubo conlleva riesgo de obstrucción, sobre todo cuando el diámetro interno es pequeño. El riesgo se reduce con el taponamiento continuo de la prótesis y la aspiración apropiada. ${ }^{13}$

Una de las complicaciones posquirúrgicas más frecuentes es el granuloma del borde superior del tubo en $\mathrm{T}$, por el roce de las impurezas de sus bordes con las estructuras laríngeas supraglóticas. El seguimiento endoscópico con resección del tejido de granulación es útil para resolver esta complicación.

Es fundamental prevenir la estenosis. La intubación debe realizarse con un tubo endotraqueal de tamaño apropiado, que es el de menor tamaño que permite la ventilación adecuada. Es importante mantener una sedación adecuada para evitar que el movimiento del tubo lesione las estructuras laríngeas, y se debe realizar el tratamiento del reflujo gastroesofágico y de los factores que ocasionan hipoperfusión y exacerban el trauma causado por el tubo. ${ }^{14} \mathrm{El}$ fracaso de la extubación o la disfonía persistente posterior a la extubación requiere una evaluación endoscópica a fin de prevenir la estenosis posintubación. ${ }^{1,2}$

El abordaje de la estenosis debe afrontarse de manera multidisciplinaria, y contar con un equipo formado por pediatras, neonatólogos, otorrinolaringólogos, cirujanos infantiles, neumólogos, gastroenterólogos, kinesiólogos, enfermeras y terapeutas de la voz y la deglución, teniendo cada uno, en algún momento del tratamiento, mayor o menor participación, hasta lograr los objetivos definitivos que son ventilación, deglución y voz adecuadas. ${ }^{5,15}$ 
La técnica de reconstrucción laringotraqueal ha demostrado ser un tratamiento eficaz en el manejo de la estenosis laríngea en los niños. Si bien nuestra casuística es limitada, el resultado ha sido alentador. Esperamos incrementar nuestra experiencia en esta técnica, para lograr una exitosa restauración de la vía aérea en un mayor número de pacientes.

\section{BIBLIOGRAFÍA}

1. Rodríguez H, Cuestas G, Botto H, Cocciaglia A, et al. Estenosis subglótica post-intubación en niños. Diagnóstico, tratamiento y prevención de las estenosis moderadas y severas. Acta Otorrinolaringol Esp 2013. En prensa.

2. Monnier P. Acquired post-intubation and tracheostomyrelated stenoses. En: Monnier P. Pediatric Airway Surgery. Lausanne, Switzerland: Springer; 2011. págs.183-98.

3. George M, Jaquet Y, Ikonomidis C, Monnier P. Management of severe pediatric subglottic stenosis with glottic involvement. J Thorac Cardiovasc Surg 2010;139(2):411-7.

4. Lusk R, Wolley A, Hollinger L. Laryngotracheal stenosis. En:Holinger L, Lusk R, Green C. Pediatric laryngology and bronchoesophagology. Philadelphia: Lippincott- Raven Publishers; 1997. págs.165-86.

5. Zanetta A, Tiscornia C, Rodríguez H, Cuestas G. Estenosis subglótica: Reconstrucción laringotraqueal con injerto de cartílago posterior en niños. FASO 2010; 17(1): 33-8.
6. Jaquet $\mathrm{Y}$, George M, Monnier P. Management of severe glotto-subglottic stenosis in children. Laryngoscope 2012; 122 (Suppl 4):S75-6.

7. Rodríguez H. Patología estructural de laringe y tráquea. En Macri C, Teper A. Enfermedades respiratorias pediátricas. México: Mc Graw Hill Interamericana, 2003. Págs. 351-60.

8. Cotton RT. Tratamiento de estenosis subglótica. En: Jong A, Kuppersmith R. Clínicas otorrinolaringológicas de Norteamérica. Actualización en vías respiratorias deniños. México: Mc Graw-Hill Interamerica; 2000. Págs.107-25.

9. Zanetta A, Rodríguez H, Botto H, Nieto M, et al Estenosis laríngea en pediatría. Reconstrucción laringotraqueal como tratamiento. An Otorrinolaringol Perú 2012; 17: 77-7.

10. Zanetta A, Cuestas G, Rodríguez H, Tiscornia C. Nuevo stent laríngeo en el tratamiento de la estenosis subglótica en niños. Acta Otorrinolaringol Esp 2012. http://dx.doi. org/10.1016/j.otorri.2012.05.008.

11. Monnier P. Airway stenting with the LT-Mold: experience in 30 pediatric cases. Int J Pediatric Otorhinolaryngol 2007; 71:1351-9.

12. Preciado D. Stenting in pediatric airway reconstruction. Laryngoscope 2012; 122 (Suppl 4):S97-8.

13. Phillips PS, Kubba H, Hartley BE, Albert DM. The use of the Montgomery T-tube in difficult paediatric airways. Int J Pediatr Otorhinolaryngol 2006; 70:39-44.

14. WeiJL, Bond J.Management and prevention of endotracheal intubation injury in neonates. Curr Opin Otolaryngol Head Neck Surg 2011; 19(6):474-7.

15. Zanetta A, Tiscornia $\mathrm{C}$, Rodríguez $\mathrm{H}$, Botto $\mathrm{H}$, et al. Resolución quirúrgica de las estenosis laringotraqueales en pediatría. FASO; 2011: 64-71. 